\title{
MULTIPLE SCLEROSIS AND THYROID GLAND DISEASES
}

\author{
Kalina Drenska ${ }^{1}$, Ivan Dimitrov ${ }^{2}$, Alexandra Tsoukeva ${ }^{1}$, Ara Kaprelyan ${ }^{1}$, \\ Tihomir Drenski ${ }^{3}$, Violeta Staneva ${ }^{4}$
}

\author{
${ }^{1}$ Department of Neurology and Neuroscience, Medical University of Varna, First Clinic of \\ Neurology, St. Marina University Hospital, Varna, Bulgaria \\ ${ }^{2}$ Department of Nursing, Sliven Affiliate, Medical University of Varna, First Clinic of \\ Neurology, St. Marina University Hospital, Varna, Bulgaria \\ ${ }^{3}$ Department of Intensive Treatment of Neurological Diseases, St. Marina University \\ Hospital, Varna, Bulgaria \\ ${ }^{4}$ Chief nurse, St. Marina University Hospital, Varna, Bulgaria
}

\begin{abstract}
Multiple sclerosis (MS) often presents with accompanying diseases. Thyroid gland pathology is not a rare finding in MS patients. Among a hospital cohort of 335 MS patients, 16 females with five thyroid gland diseases were diagnosed during a 14 -year period. There were six cases of diffuse goiter, four of postoperative hypothyroidism, three of thyroid adenoma, two of autoimmune thyroiditis, and one of hyperthyroidism. The age characteristics of the patients varied for the specific diseases. We emphasize the need for regular monitoring of the thyroid function in MS patients.
\end{abstract}

Keywords: multiple sclerosis, diffuse goiter, autoimmune thyroiditis, thyroid adenoma, postoperative hypothyroidism, hyperthyroidism, comorbidity

\section{INTRODUCTION}

Multiple sclerosis (MS) is a severe demyelinating disease of the central nervous system resulting in gradual disability. It mainly affects the most active age, between 20 and 40 years. Thyroid gland pathology occurs more commonly in females and usually necessitates specific lifelong drug treatment. Recent investigations indicate the co-occurrence of MS with several thyroid gland diseases, suggesting the mutual aggravation of the symptoms of these disorders.

Address for correspondence:

Kalina Drenska, $M D$

First Clinic of Neurology

Medical University of Varna

St. Marina University Hospital of Varna

1 Hristo Smirnenski Str., 9010 Varna, Bulgaria

e-mail:k_drenska@abv.bg

Received: April 18, 2016

Accepted: May 11, 2016

\section{MATERIALS AND METHODS}

Our study covered a total of 16 female patients with MS and accompanying thyroid gland diseases at a mean age of $48.6 \pm 10.3$ years (range 23 to 71 years). They were hospitalized in the First Clinic of Neurology and in the MS Centre at St. Marina University Hospital,Varna, during the period between January 1, 2002 and December 31, 2015. The total number of MS patients was 305, i.e. the prevalence of thyroid comorbidity reached $5.25 \%$.

The retrospective analysis of these 16 patients included the following parameters: age at the most recent hospitalization; initial age of MS, initial age of thyroid disease (diffuse goiter, postoperative hypothyroidism, thyroid adenoma, autoimmune or Hashimoto's thyroiditis, and hyperthyroidism or Graves' disease), number of MS attacks since the onset of MS, order of occurrence of MS and thyroid disease, and examinations of thyroid hormones (TSH, free T3, free T4), and anti-TPO. 
Kalina Drenska, Ivan Dimitrov, Alexandra Tsoukeva et al.

\section{RESULTS AND DISCUSSION}

Table 1 demonstrates the age and time-interval distributions of the patients with MS and thyroid gland diseases. There are significant differences between the patients with MS and thyroid gland diseases concerning their age range at onset and combined duration of two diseases, number of MS attacks and of years per MS attack. in one patient - up to the twelfth year of the detected period.

Our results shed light on some features of this comorbidity and provide evidence of the need for regular monitoring of the thyroid function in MS patients. It is noteworthy that, in most cases, the recent values of thyroid hormones were within normal limits.

Table 1. Age and time-interval distributions of the patients with MS and thyroid gland diseases

\begin{tabular}{|c|c|c|c|c|c|c|}
\hline Thyroid disease & $\mathrm{n}$ & $\begin{array}{c}\text { Age range at } \\
\text { hospitalization }\end{array}$ & $\begin{array}{l}\text { Age range at } \\
\text { MS onset }\end{array}$ & $\begin{array}{l}\text { Age at thyroid } \\
\text { disease onset }\end{array}$ & MS attacks & $\begin{array}{l}\text { Range of years } \\
\text { per MS attack }\end{array}$ \\
\hline diffuse goiter & 6 & $41-71$ & $32-55$ & $25-61$ & $1-9$ & $1.22-13$ \\
\hline $\begin{array}{l}\text { postoperative hypothy- } \\
\text { roidism }\end{array}$ & 4 & $42-55$ & $29-47$ & $31-44$ & $3-5$ & $1.33-4.33$ \\
\hline thyroid adenoma & 3 & $50-61$ & $41-46$ & $37-51$ & $3-6$ & $1.67-3$ \\
\hline autoimmune thyroiditis & 2 & 23,46 & 22,34 & 16,38 & 1,6 & 1,2 \\
\hline hyperthyroidism & 1 & 43 & 32 & 28 & 3 & 3.67 \\
\hline
\end{tabular}

We have found that the number of patients in whom MS precedes the thyroid gland disease is eight, and, vice versa, in whom the thyroid gland disease precedes MS by certain number of years is seven. In one patient both MS and postoperative hypothyroidism occurred in the same year.

patients

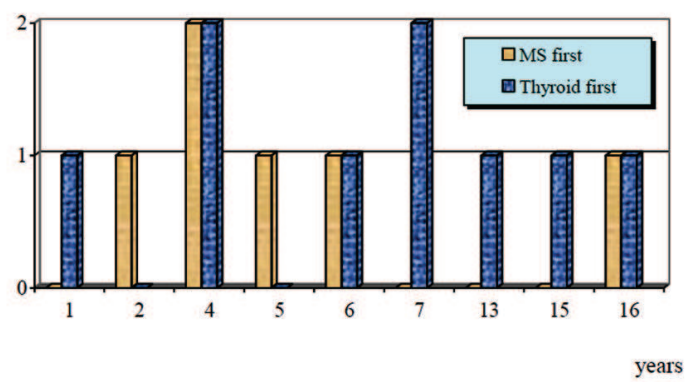

Fig. 1. Number of patients in whom MS or thyroid gland disease occur first by number of years

We have detected the number of patients with simultaneous occurrence of MS and a thyroid gland disease during the different years of observation. Both diseases were coexisting up to the tenth year in ten patients, up to the fifth year in eleven ones and
Several studies have evaluated the occurrence of autoimmune thyroid disease in MS population, reporting prevalences ranging from 0.92 to $13.9 \%$ (1). In 2005, the age-adjusted prevalence of autoimmune thyroid disease in the province of Manitoba, Cana$\mathrm{da}$, is $9.51 \%$ (95\% CI 8.46-10.6) in the MS population and $8.56 \%$ (95\% CI 8.11-9.02) in the general one. Its age-adjusted incidence per 100000 persons per year is 422.8 (95\% CI 204.4-641.3) in the MS population and 407.7 (95\% CI 308.5-506.9) in the general one (2).

MS, Hashimoto's disease and Graves' disease are autoimmune diseases that may share similar pathogenic mechanisms. There is a significant co-occurrence of Graves' disease ( $\mathrm{p}=0.002)$ and Hashimopatients

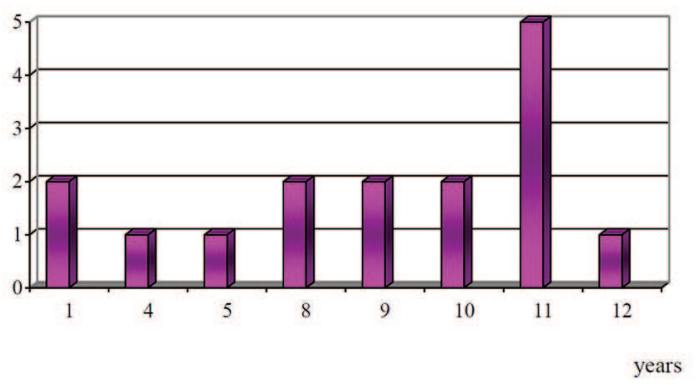

Fig. 2. Number of patients with co-occurrence of MS and a thyroid gland disease in different years 
to's disease $(\mathrm{p}=0.097)$ with MS in Newfoundland and Labrador, Canada (3). There are insignificant differences between the age of onset of all MS patients and those with both Graves' $(\mathrm{p}=0.81)$ and Hashimoto's $(\mathrm{p}=0.71)$ disease (3).

Interferon- $\beta$ but not glatiramer acetate stimulates CXCL10 secretion in primary cultures of thyrocytes. Significant and dose-dependent secretions of CXCL10 are induced by interferon- $\beta$ but not by glatiramer acetate. These results could explain the occurrence of autoimmune thyroid disease during the treatment of MS by interferon- $\beta$ but not by glatiramer acetate (4).

Proximal muscle weakness, myalgia, and fatigue are common symptoms in MS and hypothyroidism.

Hypothyroidism caused by Hashimoto thyroiditis displays a tendency to be more severe and more common in MS patients (5).

\section{CONCLUSION}

There is accumulating evidence that thyroid pathology should be taken in consideration in the differential diagnosis of several systemic and autoimmune diseases and especially in MS patients, although the clinical diagnosis of thyroid gland disorders is less common than their laboratory verification. The thyroid pathology of MS patients is probably determined by the conducted therapy and etiopathogenetic factors underlying disease.

\section{REFERENCES}

1. Seyfert, S., Klapps, P., Meisel, C., Fischer, T. and Junghan, U., Multiple sclerosis and other immunologic diseases. Acta Neurol Scand, 81: 37-42, 1990.

2. Marrie, R.A., Yu, B.N., Leung, S., Elliott, L., Warren, S., Wolfson, C., Tremlett, H., Fisk, J. and Blanchard, J., The incidence and prevalence of thyroid disease do not differ in the multiple sclerosis and general populations: a validation study using administrative data. Neuroepidemiology, 39: 135142, 2012.

3. Sloka, J.S., Phillips, P.W., Stefanelli, M. and Joyce, C., Co-occurrence of autoimmune thyroid disease in a multiple sclerosis cohort. J Autoimmune Dis, 2: 9, 2005 Nov 9.

4. Rotondi, M., Stufano, F., Lagonigro, M.S., La Manna, L., Zerbini, F., Ghilotti, S., Pagliari, M. T., Coperchini, F., Magri, F., Bergamaschi, R., Oliviero,
A. and Chiovato, L., Interferon- $\beta$ but not glatiramer acetate stimulates CXCL10 secretion in primary cultures of thyrocytes: a clue for understanding the different risks of thyroid dysfunctions in patients with multiple sclerosis treated with either of the two drugs. J Neuroimmunol, 234: 161-264, 2011.

5. Niederwieser, G., Buchinger, W., Bonelli, R.M., Berghold, A., Reisecker, F., Költringer, P. and Archelos, J.J., Prevalence of autoimmune thyroiditis and non-immune thyroid disease in multiple sclerosis. J Neurol, 250: 672-675, 2003. 